\title{
Adesão dos idosos cadastrados no Projeto 'Faculdade Integrada da Terceira Idade' frente à hipertensão arterial
}

Existem vários conceitos que irão presidir a forma de o indivíduo enfrentar o processo de envelhecer bem, como compreender todas as mudanças existentes durante esse evento. $\mathrm{O}$ trabalh visa identificar a incidência de idosas hipertensas participantes do Projeto 'Faculdade Integrada da Terceira Idade' (FINATI) existente na Universidade UniAGES. Esse trabalho é um estudo descritivo, exploratório, transversal, de campo, com abordagem quantitativa e qualitativa. O trabalho foi aprovado pelo Comitê de Ética e Pesquisa da Faculdade de Ciências Humanas e Sociais, sob parecer de número 850215 . Em relação aos hábitos e estilo de vida, 76,7\% informou não fazer uso de bebida alcoólica; e 66,7\% não possui o habito de fumar. As idosas participantes do estudo classificaram o seu estado de como bom ( $23,3 \%)$; regular $(76,7 \%)$. Dores nas costas $(90 \%)$ e a presença de varizes $(60 \%)$ foram as condições de saúde mais presentes
no histórico pessoal dessas idosas além a HAS como já foi referido. Dentre as 29 idosas hipertensas, $(n=23 ; 79,3 \%$ ) afirmam ter interrompido o tratamento em algum momento do curso terapêutico em contra partida das ( $n=6 ; 20,7 \%)$ que assumem não ter interrompido a dinâmica terapêutica; remédios muito caros $(n=18 ; 62,1 \%) ;$ indisponibilidade nas farmácias, UBS hospitais $(n=25 ; 86,2 \%)$; assintomatologia da doença $(n=7 ; 24,1 \%)$ e esquecimento $(n=23 ; 79,3 \%)$ foram as variáveis mais relevantes que justificou a interrupção do tratamento medicamento na população estudada o que de certo modo provoca prejuizos para o controle da HAS. Entre os motivos pelo quais as idosas tenham faltado às consultas médicas, temos o horário de atendimento $(n=7 ; 24,1 \%)$, a insatisfação pelo profissional que as atende $(n=9 ; 31,0 \%)$, o tempo de espera pelas consultas $(n=9 ; 31,0 \%)$ e as consultas muito rápidas $(n=41,4 \%)$. Desse modo, fomenta-se a necessidade de uma consciência entre a prescrição médica e o comportamento adotado pelo paciente, o que não afasta a participação efetiva dos familiares e de toda a equipe de saúde frente às mudanças que serão necessárias no decorrer do plano terapêutico.

Palavras-chave: Enfermagem; Hipertensão Arterial Sistêmica; Adesão; Tratamento.

\section{Adherence of the elderly registered in the Project 'Integrated College of the Third Age' facing hypertension}

\begin{abstract}
There are several concepts that will govern how an individual face the aging process well, such as understanding all the changes that occur during this event. The work aims to identify the incidence of hypertensive elderly women participating in the Project 'Integrated College of the Third Age '(FINATI) existing at UniAGES University. This work is a descriptive, exploratory crosssectional field study with a quantitative and qualitative approach. The study was approved by the Ethics and Research Committee of the Faculty of Humanities and Social Sciences, under opinion number 850215 . Regarding habits and lifestyle, $76.7 \%$ reported not using alcohol; and $66.7 \%$ do not have a smoking habit. The elderly participants in the study rated their status as good $(23.3 \%)$; regular $(76.7 \%)$. Back pain $(90 \%)$ and the presence of varicose veins $(60 \%)$ were the health conditions most present in the personal history of these elderly women besides hypertension, as already mentioned. Among the 29 hypertensive elderly women $(n=23 ; 79.3 \%)$ claim to have discontinued treatment at some point in the course of treatment in contrast to those $(n=6 ; 20.7 \%)$ who assume they have not interrupted the therapeutic dynamics; very expensive medicines $(n=18 ; 62.1 \%)$; unavailability in pharmacies, BHUs and hospitals $(n=25$ $86.2 \%)$; asymptomatology of the disease $(n=7 ; 24.1 \%)$ and forgetfulness $(n=23 ; 79.3 \%)$ were the most relevant variables that justified the interruption of drug treatment in the studied population, which in some way causes damage to the control. of HAS. Among the reasons why the elderly women missed medical appointments were the hours of service $(n=7 ; 24.1 \%)$ dissatisfaction with the professionals who attend them $(n=9 \cdot 31.0 \%$, waiting for consultations $(n=9 ; 31.0 \%)$ and very fast consultations $(n=41.4 \%)$. Thus, it fosters the need for awareness between medical prescription and the behavior adopted by the patient, which does not preclude the effective participation of family members and the entire health team in the face of changes that will be necessary during the therapeutic plan.
\end{abstract}

Keywords: Nursing; Systemic Arterial Hypertension; Accession; Treatment.

Topic: Enfermagem em Saúde Pública

Reviewed anonymously in the process of blind peer
Received: 10/03/2019

Approved: 11/05/2019
Wellington Pereira Rodrigues (iD)

Centro Universitário AGES, Brasil http://lattes.cnpq.br/2798105983752891 http://orcid.org/0000-0002-0993-6871

wellington life@live.com

Ana Paula Gomes Soares (it)

Universidade Federal de Viçosa, Brasil http://lattes.cnpq.br/3631825469916595 http://orcid.org/0000-0002-1050-6035 anapaulagsoares@yahoo.com.br

Priscila Dantas Gonçalves (iD Centro Universitário AGES, Brasil http://lattes.cnpq.br/7078066094809493 http://orcid.org/0000-0002-1849-0226 prisciladantasg@hotmail.com

\author{
Elisângela Oliveira de Carvalho (iD) \\ Universidade Federal do Amazonas, Brasil \\ http://lattes.cnpq.br/1752425604850531 \\ http://orcid.org/0000-0002-8252-1437 \\ eli@gmail.com \\ Leonardo Fabrício Gomes Soares (iD) \\ Universidade Federal de Minas Gerais, Brasil \\ http://lattes.cnpq.br/837835063298930 \\ http://orcid.org/0000-0002-4043-5557 \\ renansallazar@gmail.com \\ Cinthia Caldas Rios Soares \\ Centro Universitário de Muriaé, Brasil \\ http://lattes.cnpq.br/7524534278738838 \\ http://orcid.org/0000-0001-5596-0007 \\ renansallazar@gmail.com
}

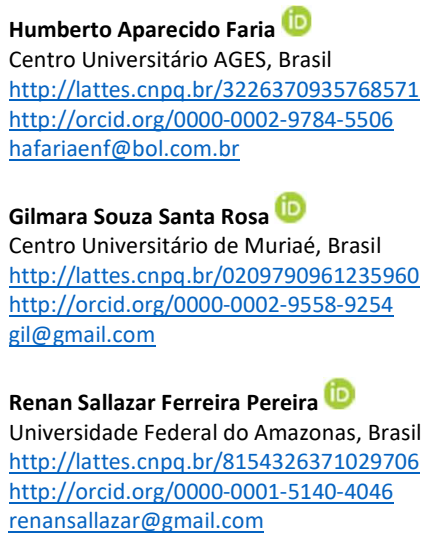

Humberto Aparecido Faria (iD)

Centro Universitário AGES, Brasi http://lattes.cnpq.br/3226370935768571

Centro Universitário de Muriaé, Brasil gil@gmail.com

Renan Sallazar Ferreira Pereira http://lattes.cnpq. br/8154326371029706 http://orcid.org/0000-0001-5140-4046 c

DOI: $10.6008 / C B P C 2236-9600.2019 .002 .0004$
Referencing this:

RODRIGUES, W. P.; SOARES, A. P. G.; GONÇALVES, P. D.; CARVALHO, E. O.; SOARES, L. F. G.; SOARES, C. C. R.; FARIA, H. A.; ROSA, G. S. S.; PEREIRA, R. S. F.. Adesão dos idosos cadastrados no Projeto 'Faculdade Integrada da Terceira Idade' frente à hipertensão arterial. Scire Salutis, v.9, n.2, p.27-36, 2019. DOI:

http://doi.org/10.6008/CBPC2236-9600.2019.002.0004 


\section{INTRODUÇÃO}

O trabalho apresenta uma fundamental relevância frente à sociedade e a maneira pela qual as pessoas que nela estão inseridas enfrentam determinadas transformações mediante o processo saúdedoença, principalmente da pessoa idosa, assim como apresenta significância para os profissionais de saúde que irão trabalhar com tais mudanças funcionais no decorrer do tempo, sendo importante estimular o envelhecimento ativo (BLOOM, 2011).

Todo ser humano atravessa processos de modificações, sendo que em cada etapa da vida ocorrem alterações das quais são vivenciadas de forma individualizada por cada indivíduo desde a infância ao envelhecer, aonde o processo de envelhecimento se trata de um fenômeno complexo e extremamente influenciado por diversos fatores que irão caracterizar um evento saudável ou não, sendo esse marcado pelo decréscimo da função fisiológica, biológica, psicológica e socioeconômica influenciados pelos fatores intrínsecos e extrínsecos do idoso sempre relacionado ao meio em que se encontra inserido e que de certo modo são responsáveis pelo aumento da incidência de problemas cardiovasculares. Contudo, o processo de envelhecimento se trata de um evento sequencial, cumulativo, irreversível e inevitável que traz consigo diversas situações e mudanças (SUELY et al., 2011).

Existem vários conceitos que irão presidir a forma do indivíduo enfrentar o processo de envelhecer bem, como compreender todas as mudanças existentes durante esse evento. Desse modo a forma em que se caracteriza o processo de envelhecimento será desmistificado por meio da compreensão sobre a diferença entre a senescência e a senilidade, norteando exatamente de que maneira o indivíduo estar envelhecendo e de que forma o processo patológico causará impacto nessa etapa da vida. Desse modo, mediante ao processo de envelhecer e as necessidades assistenciais que lhes são dirigidas, ressalta-se sempre que, cada idoso apresenta seus aspectos biopsicossociais de forma individualizada requerendo tipos de assistências diferenciadas no que tange a sua individualidade e necessidades apresentadas, sejam elas apontadas por sua senilidade e/ou senescência (BRASIL, 2010).

É notável, através de revisões literárias, que nos últimos anos os aspectos sociodemográficos voltado à população idosa vêm apresentando um crescimento relevante em função da influência da diminuição das taxas de mortalidades em idosos, o aumento da expectativa de vida desses e principalmente em função do declínio das taxas de natalidade.

Em função de tais justificativas, o número de idosos com mais de 65 anos irá quadriplicar até 2060, passando de 14,9 milhões (7,4\%) para 58,4 milhões $(26,7 \%)$, o que, por sua vez, provoca mudanças não só na situação demográfica e estrutura etária dessa população como também promove alterações na situação epidemiológica da população de idosos como um todo e uma série de desafios para o Sistema Único de Saúde (SUS) e Políticas Públicas frente às Doenças Crônicas Não Transmissíveis (DCNT) uma vez que tais doenças são decorrentes do processo de decréscimo fisiológico, biológico, bioquímico e morfológico vivenciados pelo idoso (IBGE, 2018). 
Como exemplo das doenças que acometem os idosos, a alta prevalência da HAS vem se destacando. Essa condição se trata de um problema de Saúde Pública intimamente ligada às taxas de mortalidade decorrentes de doenças cardiovasculares influenciada pela HAS descompensada ou não tratadas adequadamente. Essas necessitam prioritariamente do desenvolvimento e atuação de Políticas Públicas de Saúde e processos de educação permanente proporcionada pelas equipes de profissionais com o foco voltado a população idosa com problemas dessa natureza de modo a minimizar o surgimento de novos casos e problemas subsequentes (IBGE, 2010).

Devido à sua alta prevalência e sua íntima relação com as doenças cardiovasculares, a HAS ainda se trata de uma situação modificável revelando-se uma necessidade emergente por parte dos idosos no que diz respeito à adesão do seu tratamento adequado de modo a minimizar e/ou erradicar danos subsequentes dos quais não somente atinge a população idosa como também as taxas orçamentárias do país referente aos gastos com hospitalizações de longas permanências e complexas em decorrência e consequência da Hipertensão Arterial Sistêmica não tratada adequadamente (BRASIL, 2011).

Vários estudos têm contribuído frente à reflexão acerca da prevenção de complicações advindas das HAS, sendo o acompanhamento medicamentoso concomitante à mudança no estilo de vida às condutas mais resolutivas. Tais condutas ascendem à necessidade de o paciente tornar-se protagonista da sua saúde e aderir adequadamente ao tratamento anti-hipertensivo (LIBERMAN, 2007).

Assim, este estudo tem por objetivos identificar a incidência de idosas hipertensas participantes do Projeto Faculdade Integradas da Terceira Idade (FINATI), bem como identificar os fatores de risco para o surgimento da HAS, verificar as variáveis que interferem na terapia medicamentosa, identificar o conhecimento das idosas frente à importância de aderir ao tratamento, os riscos da não adesão à terapêutica e a percepção dessas idosas quanto às características da doença e seus fatores de risco.

Mediante revisões bibliográficas e a pesquisa de campo da qual foi realizada frente ao processo de adesão a terapêutica anti-hipertensiva, observaram-se que as queixas voltadas a não adesão adequada ao tratamento apresentou relevância frente ao esquecimento quanto à tomada das medicações e dificuldades das idosas associarem o horário da medicação com alguma atividade diária da qual caracteriza a dificuldade terapêutica sequencial o que de fato acaba por prejudicar o ciclo das medicações. Relatos sobre os altos preços das medicações juntamente a falta dos mesmos nos postos de saúde e farmácias populares se destacaram com relevância. A assintomatologia característica da HAS também se destacou como variável que impossibilita a adesão terapêutica de forma adequada, seguido da insatisfação do paciente para com o profissional que os atente em consultas muitos rápidas (LIBERMAN, 2007).

\section{METODOLOGIA}

Trata-se de um estudo com características descritivas, exploratório, transversal, de campo com abordagem quantitativa e qualitativa, realizado com 30 idosas cadastradas no Projeto FINATI que participaram do estudo sobre 'Adesão ao Tratamento da Hipertensão Arterial Sistêmica dos Idosos 
Cadastrados no Programa do FINATI' cujo objetivo geral foi analisar o entendimento e a percepção dos portadores de Hipertensão Arterial do Programa FINATI acerca da doença e da importância da sua adesão ao tratamento e os riscos da não adesão terapêutica; esse estudo foi realizado no Projeto Faculdade Integrada da Terceira Idade FINATI existente na Universidade UniAGES localizada no Município de Paripiranga-Ba, aprovado pelo Comitê de Ética e Pesquisa da Faculdade de Ciências Humanas e Sociais, sob parecer de número 850215 . A coleta de dados procedeu por meio de questionários direcionados as Idosas participantes do FINATI durante os dias em as mesmas desenvolvem suas atividades físicas.

O questionário apresentou variáveis selecionadas para o posterior desenvolvimento do estudo como: dados pessoais (idade, sexo, etnia, escolaridade, estado civil, naturalidade e cidade onde mora); dados sobre o estilo de vida (ingestão de bebida alcoólica, hábito de fumar, prática de exercícios físicos e regularidades e hábitos alimentares); morbidades referidas em geral e direcionadas a HAS; uso de medicamentos e sua frequência; vulnerabilidade ao estresse; avaliação quanto ao nível de conhecimento relacionado à doença; razões de não adesão ao tratamento; atitudes para a tomada das medicações; frequência nas consultas médicas. Os dados foram armazenados em banco de dados e confeccionados por meio de planilhas no Excel onde foram analisados quantitativamente e apresentados em forma de tabelas.

\section{RESULTADOS}

Mediante à amostra estudada nota-se que todas são do sexo feminino (100\%) de etnia branca (76,7\%); apresentou faixa etária de 60-70 anos (66,7\%) sendo a idade mínima 60 e a idade máxima 90 anos; para as idosas casadas ou amasiadas totalizou um percentil de (63,3\%); na escolaridade foram identificadas (23,3\%) para as analfabetas e (26,7\%) para aquelas com nível primário completo.

Em relação aos hábitos e estilo de vida, $(76,7 \%)$ referiu não fazer uso de bebida alcoólica; $(66,7 \%)$ não possui o habito de fumar. Todas praticam exercícios físicos (100\%) em até duas vezes por semana (63,3\%), ressaltando que utilizam o FINATI como única modalidade para a prática da atividade física. Referente ao habito alimentar, (50\%) relatam fazer uso de alimentos naturais concomitantes ao alimento processado.

Sua forma de ingestão foi representada por $(96,7 \%)$ para os alimentos cozidos; $(87,7 \%)$ dessa amostra não adicionam o sal no preparo dos alimentos embora (60\%) dessas utilizam o tempero industrializado no preparo dos alimentos do qual possui um percentual de sódio acima do que é permitido e recomendado pela Sociedade Brasileira de Cardiologia, da qual se recomenda apenas $6 \mathrm{~g}$ de sódio diariamente. Dosagem essa não atribuída aos alimentos processados dos quais possuem um volume maior na quantidade de sódio.

As idosas participantes do estudo classificaram o seu estado de como bom (23,3\%); regular (76,7\%). Tais variáveis se apresentam como essenciais para que o indivíduo possa estabelecer a sua autopercepção e procurar por assistência de saúde para a correção do que seja necessário. Em relação ao total de participantes do estudo, das 30 idosas abordadas, $(96,7 \%)$ possuem a HAS como patologia de base. Dessas, o tempo mínimo da presença dessa condição é de 5 anos em relação ao tempo máximo de 30 anos. 
Dores nas costas $(90 \%)$ e a presença de varizes $(60 \%)$ foram às condições de saúde mais presentes no histórico pessoal dessas idosas além a HAS como já foi referido. A presença da HAS, IAM, AVC, DM e dislipidemias foram às doenças mais encontradas no histórico familiar dessas idosas o que se trata de fatores de risco para que as mesmas venham a desenvolver determinadas patologias em função do histórico familiar bem como por influência dos agravos proporcionados pela HAS não tratada adequadamente. Nessa Perspectiva, apresenta-se a tabela 1.

Tabela 1: Distribuição dos idosos cadastrados no FINATI em relação ao conhecimento da doença em 2018.

\begin{tabular}{|c|c|c|c|c|}
\hline VARIÁVEIS & $\mathbf{N}$ & $\%$ & $\mathbf{N}$ & $\%$ \\
\hline \multicolumn{5}{|l|}{ Perguntas Sobre o Conhecimento da Doença. } \\
\hline 1. Pressão alta é para toda a vida? & 18 & 60,0 & 12 & 40,0 \\
\hline 2. Pressão alta é assintomática? & 15 & 50,0 & 15 & 50,0 \\
\hline 3. Pressão alta é 14 por 9 ? & 26 & 86,7 & 4 & 13,3 \\
\hline 4. Pressão alta traz complicações? & 22 & 73,3 & 8 & 26,7 \\
\hline 5. O tratamento é para a vida toda? & 16 & 53,3 & 14 & 46,7 \\
\hline 6. Pressão alta pode ser tratada? & 13 & 43,3 & 17 & 56,7 \\
\hline 7. Fazer exercícios físicos ajuda? & 26 & 86,7 & 4 & 13,3 \\
\hline 8. Perder peso ajuda a controlar a pressão arterial? & 29 & 96,7 & 1 & 3,3 \\
\hline 9. Diminuir o sal ajuda a controlara pressão? & 30 & 100 & $\ldots$ & $\ldots$ \\
\hline 10. Diminuir o nervosismo ajuda a controlar a pressão arterial? & 23 & 76,7 & 7 & 23,3 \\
\hline
\end{tabular}

Verificou-se aqui que o nível de conhecimento sobre a doença foi satisfatório nos pacientes participantes da pesquisa. O percentual de valores para as respostas verdadeiras são superiores aquelas com respostas negativas. Mediante a relação de perguntas verificou-se que grande parte das idosas entrevistadas possui um nível de conhecimento satisfatório em relação à doença, sua forma de tratamento e medidas para o seu controle, mas esse fato não determina que a adesão ao tratamento seja adequada. Para justificar os motivos pela interrupção do tratamento, os dados foram agrupados na tabela 2.

Tabela 2: Distribuição dos idosos cadastrados no FINATI frente às razões de não adesão ao tratamento em 2018.

\begin{tabular}{|c|c|c|}
\hline VARIÁVEIS & $\mathbf{N}$ & $\%$ \\
\hline \multicolumn{3}{|l|}{ Interrompeu o Tratamento } \\
\hline Sim & 23 & 79,3 \\
\hline Não & 6 & 20,7 \\
\hline \multicolumn{3}{|l|}{ Motivos } \\
\hline 1. Remédios muito caros. & 18 & 62,1 \\
\hline 2. Não há na farmácia nem no hospital. & 25 & 86,2 \\
\hline 3. Ter que tomar várias vezes ao dia. & 1 & 3,4 \\
\hline 4. Não sente nada. & 7 & 24,1 \\
\hline 5. Não sente necessidade de tratar. & 1 & 3,4 \\
\hline 6. Acha que estar curado. & 1 & 3,4 \\
\hline 7. Hipertensão não é tão grave. & 1 & 3,4 \\
\hline 8. Tomar apenas quando se sente mal. & 3 & 10,3 \\
\hline \multicolumn{3}{|c|}{ 9. Falta de orientação quanto ao uso dos medicamentos. } \\
\hline 10. Esquecimento & 23 & 79,3 \\
\hline
\end{tabular}

Dentre as 29 idosas hipertensas, ( $n=23 ; 79,3 \%$ ) afirmam ter interrompido o tratamento em algum momento do curso terapêutico em contra partida das $(n=6 ; 20,7 \%)$ que assumem não ter interrompido a dinâmica terapêutica; remédios muito caros $(n=18 ; 62,1 \%)$; indisponibilidade nas farmácias, UBS e hospitais ( $n=25 ; 86,2 \%)$; assintomatologia da doença $(n=7 ; 24,1 \%)$ e esquecimento $(n=23 ; 79,3 \%)$ foram as variáveis mais relevantes que justificou a interrupção do tratamento medicamento na população estudada o que de certo modo provoca prejuízos para o controle da HAS. 
Tabela 3: Distribuição dos idosos cadastrados no FINATI frente às razões da falta nas consultas médicas em 2018.

\begin{tabular}{|c|c|c|}
\hline VARIÁVEIS & $\mathbf{N}$ & $\%$ \\
\hline \multicolumn{3}{|l|}{ Falta na Consulta Médica } \\
\hline Sim & 10 & 34,5 \\
\hline Não & 19 & 65,5 \\
\hline \multicolumn{3}{|l|}{ Motivos } \\
\hline \multicolumn{3}{|l|}{ 1. Esquecimento } \\
\hline 2. Dinheiro para condução & 3 & 10,3 \\
\hline \multicolumn{3}{|l|}{ 3. Deixar os filhos } \\
\hline 4. Viagens & 1 & 3,4 \\
\hline 5. Sentia-se bem & 4 & 13,8 \\
\hline 6. Horário de atendimento & 7 & 24,1 \\
\hline \multicolumn{3}{|l|}{ 7. Necessidade de companhia } \\
\hline 8. Não gostou do médico & 9 & 31,0 \\
\hline \multicolumn{3}{|l|}{ 9. Distância } \\
\hline \multicolumn{3}{|l|}{ 10. Não viu necessidade de tratar. } \\
\hline 11. Tempo de espera. & 9 & 31,0 \\
\hline \multicolumn{3}{|l|}{ 12. Para não faltar ao trabalho. } \\
\hline \multicolumn{3}{|l|}{ 13. Mal informado quanto ao retorno. } \\
\hline 14. Consulta muito rápida. & 12 & 41,4 \\
\hline
\end{tabular}

Entre os motivos pelo quais as idosas tenham faltado às consultas médicas temos o horário de atendimento $(n=7 ; 24,1 \%)$, a insatisfação pelo profissional que as atende $(n=9 ; 31,0 \%)$, o tempo de espera pelas consultas ( $n=9 ; 31,0 \%)$ e as consultas muito rápidas $(n=41,4 \%)$. Tais motivos se apresentaram como variáveis relevantes que justificou a não presença constante das idosas nas consultas médica da qual é representada por $(n=10 ; 34,5 \%)$ do total da população estudada.

Tabela 4: Distribuição dos idosos cadastrados no FINATI frente à tomada de remédios em 2018.

\begin{tabular}{|c|c|c|}
\hline VARIÁVEIS & $\mathbf{N}$ & $\%$ \\
\hline \multicolumn{3}{|c|}{ 1. Anota horário } \\
\hline Sim & 6 & 20,7 \\
\hline Não & 23 & 79,3 \\
\hline \multicolumn{3}{|c|}{ 2. Toma no mesmo horário } \\
\hline Sim & 7 & 24,1 \\
\hline Não & 22 & 75,9 \\
\hline \multicolumn{3}{|c|}{ 3. Associa com alguma atividade } \\
\hline Sim & 7 & 24,1 \\
\hline Não & 22 & 75,9 \\
\hline \multicolumn{3}{|c|}{ 4. Toma a medicação antes de sair de casa } \\
\hline Sim & 18 & 62,1 \\
\hline Não & 11 & 37,9 \\
\hline \multicolumn{3}{|c|}{ 5. Providencia outra caixa antes de acabar } \\
\hline Sim & 27 & 93,1 \\
\hline Não & 2 & 6,9 \\
\hline \multicolumn{3}{|c|}{ 6. Leva a medicação quando viaja } \\
\hline Sim & 29 & 100 \\
\hline \multicolumn{3}{|l|}{ Não } \\
\hline \multicolumn{3}{|c|}{ 7. Toma com a pressão controlada } \\
\hline Sim & 18 & 62,1 \\
\hline Não & 11 & 37,9 \\
\hline \multicolumn{3}{|c|}{ 8. Deixa de tomar quando ingere bebida alcoólica } \\
\hline Sim & 6 & 20,7 \\
\hline Não & 23 & 79,3 \\
\hline \multicolumn{3}{|c|}{ 9. Deixou de tomar nos últimos $\mathbf{3 0}$ dias } \\
\hline Sim & 16 & 55,2 \\
\hline Não & 13 & 44,8 \\
\hline \multicolumn{3}{|c|}{ 10. Faltou à consulta nos últimos 6 meses } \\
\hline Sim & 11 & 37,9 \\
\hline Não & 18 & 62,1 \\
\hline
\end{tabular}

Mediante à fidelidade quanto à tomada das medicações, nota-se um comportamento inadequado das idosas frente à dinâmica terapêutica. As situações mais referidas, frente à tomada das medicações foram justificadas por ( $n=23 ; 79,3 \%$ ), para o esquecimento das idosas quanto ao anotar o horário de ingeri-los; 
( $n=22 ; 75,9 \%$ ) não utilizam a medicação nos mesmos horários correspondente ao uso adequado, percentual esse que também representa a população que não associa os horários de uso com alguma atividade como forma de lembrete; $(n=18 ; 62,1 \%)$ da população estudada tomam a medicação antes de sair de casa; ( $n=27$; $93,1 \%)$ providencia oura caixa; $(n=29 ; 100 \%)$ levam a medicação antes de sair de casa. Medida essa que não irá garantir que o seu uso será adequado; $(n=18 ; 62,1 \%)$ dessa população faz uso das medicações mesmo quando esta se encontra controlada; $(n=23 ; 79,3 \%)$ não deixam de usar a medicação quando ingere bebida alcoólica.

Essa população que relata não deixar de usar a medicação é a mesma que informa durante a pesquisa, não fazer uso de bebidas alcoólicas. Dessa forma não tem por que suspender o uso da terapia medicamentosa; ( $n=16 ; 55,2 \%)$ deixou de tomar as medicações nos últimos 30 dias e ( $n=18 ; 62,1 \%)$ dessas relatou faltar nas consultas médicas nos últimos seis meses. Tais informações se tratam de respostas das idosas obtidas frente as suas atitudes em relação à tomada das medicações que por sua vez não demonstram fidelidade quanto à adesão ao tratamento anti-hipertensivo.

\section{DISCUSSÃO}

Os resultados obtidos apontam que não houve representação masculina no grupo pesquisado, destacando uma alta prevalência de mulheres hipertensas assim como evidencia a maior presença das mulheres em participações referente às atividades físicas. Estudos realizados apontam a alta prevalência das mulheres diagnosticadas com a HAS demonstrada em um percentual de $(25,4 \%)$ a mais do que os $(19,5 \%)$ que surgem na população masculina. Esse diagnóstico é justificado mediante a procura das mulheres aos serviços de saúde bem como pela oferta de Políticas Públicas ofertadas para essa população (BRASIL, 2013).

Idades entre 60 e 70 anos representou a faixa etária da população estudada da qual caracteriza o início do processo de envelhecimento e junto a esse processo os decréscimos fisiológicos e complicações subsequentes (BOCCHI et al., 2012). O estado civil se trata de uma variável que interfere diretamente no processo de diagnóstico e de adesão e sequência do tratamento anti-hipertensivo. No que se refere ao nível de escolaridade, esse se encontra como um dos fatores influentes para a adesão ao tratamento e que, o baixo nível de escolaridade se trata de um fator de risco agravante que vem a contribuir para a resistência dos pacientes em relação à adesão terapêutica seja essa medicamentosa ou comportamental (BRASIL, 2014).

Em relação aos hábitos e estilo de vida, observou-se que as participantes não possuem o hábito de ingerir bebida alcoólica e tabagismo. Tais hábitos aumentam as consequências de complicações advindas da HAS mediante o comprometimento dos vasos sanguíneos e aumento dos níveis pressóricos provocados pela nicotina e o álcool. Aconselha-se que o uso de álcool não deve ultrapassar valores de $30 \mathrm{~g}$ etanol/dia para homens e $15 \mathrm{~g}$ para mulheres (BRASIL, 2011).

A prática de exercícios físicos apresenta múltiplos benefícios e atua como hipotensor em longo prazo quando essa prática se apresenta de forma planejada e orientada (BRASIL, 2012). Tais práticas devem ser realizadas em no mínimo 30 min diários com atividades praticadas pelo menos 5 vezes por semana de forma 
continua de modo a respeitar as limitações do paciente. Esse comportamento relacionado à prática de exercícios físicos ainda não se encontra ativos conforme as orientações, uma vez que essas idosas exercem a prática somente duas vezes por semana utilizando o FINATI como única modalidade para a prática da atividade física.

Os hábitos alimentares frente ao seu preparo e consumo mostraram um potencial de risco para o agravamento da HAS uma vez que mediante o consumo de alimentos processados e a adição de temperos industrializados, as idosas tentem a ingerir uma quantidade de sódio além do recomendado que é de $6 \mathrm{~g} / \mathrm{dia}$ da qual não corresponde à dosagem existente nos temperos industrializados dos quais possuem um volume maior na quantidade de sódio (SANTOS, 2012).

O reconhecimento do seu estado de saúde se trata de um fator favorável para que as idosas participantes do estudo possam identificar a sua necessidade de busca pelo serviço de saúde e posteriormente minimizar as possibilidades de agravos a sua situação frente à doença (SBC, 2010). A quantidade de idosas hipertensas mostrou-se aumentada dentro da população estuda com tempo mínimo de 5 anos em relação ao tempo máximo de 30 anos. Nos últimos 20 anos houve uma prevalência de cerca de $30 \%$ de pessoas com HAS em toda a população brasileira assim como esse percentual vem aumentando à medida que a pessoa envelhece. Indivíduos com idade igual e acima de 60 anos a prevalência tende a aumentar em até $60,0 \%$ dos casos o que, consequentemente traz maiores riscos cardiovasculares (SOARES et al., 2012).

Tanto o histórico pessoal, quanto histórico familiar exercem influências sobre o surgimento da HAS e suas complicações. A origem de determinadas doenças crônicas apresenta a influência genética por herança familiar como uma das razões para o seu desenvolvimento, ressaltando ainda que o histórico pessoal, a maneira pela qual o paciente conduz seus comportamentos alimentares entre outros, também favorece para tais danos à saúde (MENDES, 2014). Fato esse notável na população de idosas participantes do estudo.

Verificou-se que o nível de conhecimento acerca da doença apresentou-se satisfatório mais que esse fato não determina que a adesão ao tratamento seja adequada. Vale ressaltar que, sabendo que a HAS se trata de uma condição de saúde que se apresenta de forma crônica e insidiosa, essa tende a se evoluir de forma silenciosa o que vem a contribuir de forma direta e indireta para os problemas cardiovasculares, cerebrovasculares e renais (SOARES et al., 2012). Desse modo torna-se importante que os pacientes tenham plenos conhecimentos acerca da doença e seus fatores de risco.

Por se tratar de uma condição de saúde potencialmente silenciosa, o tratamento da HAS torna-se tardio e dificultoso, assim como apresenta vários fatores e razões que impossibilitam a continuidade e fidelidade ao tratamento. Variáveis essas que foram expostas pelas idosas componentes do estudo e que necessitam de uma organização de qualidade nos serviços de saúde de modo a proporcionar um atendimento adequado pela equipe de profissionais objetivando assim melhores resultados frente à adesão ao tratamento (MENDES, 2014). 
Desse modo, vale ressaltar que a não adesão ao tratamento se trata de um forte fator de risco que vem a impedir o alcance dos objetivos terapêuticos que, consequentemente irá trazer problemas cardiovasculares aos pacientes e maiores riscos de mortes súbitas. Essa situação apresenta um grande desafio aos profissionais de saúde, e uma situação frustrante para os mesmos quando um paciente não adere ao tratamento de forma adequada (DOURADO et al., 2011).

Relacionado às questões que leva ao absenteísmo nas consultas médicas, essa apontou vários fatores que direta e indiretamente afeta na continuidade do tratamento o que proporciona uma avaliação frente à necessidade de mudar o cenário de atendimentos, uma vez que a ausência das idosas e de demais grupos populacionais nas consultas médicas pode gerar consequências irreversíveis a saúde (ABREU, 2007).

A qualidade de atendimento e da assistência prestada pelos profissionais de saúde, principalmente os médicos, trata-se de um fator bastante relevante uma vez que, quando não prestada adequadamente acaba afastando o paciente, interferindo na continuidade do seu tratamento e cuidados para com a sua saúde assim como revela o presente estudo (IBGE, 2018).

O uso contínuo das medicações se trata de uma conduta assertiva para que os níveis pressóricos se mantenham em seus níveis normais ou limítrofes segundo as condições de cada paciente. Mediante a tal explanação, nota-se um comportamento inadequado das idosas frente à dinâmica terapêutica conforme apresentam os resultados das pesquisas aonde aponta várias justificativas para explicar o motivo pelo qual as idosas não aderem corretamente ao plano terapêutico, bem como tais justificativas deverão nortear as futuras condutas dos profissionais de saúde, baseados nas Políticas Públicas de Saúde, de modo a alcançar seus objetivos acerca de diminuir a incidência de novos casos bem como minimizar as consequências súbitas e irreversíveis que estão significativamente ligadas a, HAS e a ausência de um tratamento adequado e resolutivo.

\section{CONCLUSÕES}

Mediante os resultados obtidos pode-se concluir que o principal fator para a inadequada adesão ao tratamento anti-hipertensivo foi a assintomatologia da doença, as medicações muito caras e sua indisponibilidade em hospitais, farmácias populares e UBS, seus efeitos colaterais, o esquecimento quanto ao uso das medicações, a insatisfação frente ao profissional de saúde, o tempo de espera para as consultas periódicas, consultas rápidas e os horários de atendimentos.

Conclui-se, então, que o tratamento da HAS se trata de um processo complexo e que a participação ativa do paciente é fundamental para o plano terapêutico. Desse modo, fomenta-se a necessidade de uma consciência entre a prescrição médica e o comportamento adotado pelo paciente, o que não afasta a participação efetiva dos familiares e de toda a equipe de saúde frente às mudanças que serão necessárias no decorrer do plano terapêutico. Por fim, cabe aos profissionais, mediante os programas federais voltados para saúde, criar estratégias assertivas de modo a proporcionar qualidade assistencial regado nos princípios e diretrizes do SUS, favorecendo o atendimento de acordo as peculiaridades e necessidades dos pacientes 
ofertando assim a possibilidade de enfrentar o tratamento de maneira facilitada, minimizando as adversidades existentes ao longo da vida do paciente.

\section{REFERÊNCIAS}

ABREU, R. N. D. C.; MOREIRA, T. M. M.. Fatores intervenientes na adesão ao tratamento anti-hipertensivo: avaliação dos periódicos de enfermagem de 1995 a 2005. Fortaleza: 2007.

BLOOM, D.. 7 Billion and counting. Science, v.333, p.562569, 2011.

BOCCHI, E. A.; MARCONDES-BRAGA, F. G.; BACAL, F.; FERRAZ, A. S.; ALBUQUERQUE, D.; RODRIGUES, D.. Atualização da Diretriz Brasileira de Insuficiência Cardíaca Crônica: 2012. Arquivos Brasileiros de Cardiologia, v.98, p.133, 2012.

BRASIL. Ministério da Saúde. Atenção à saúde da pessoa idosa e envelhecimento. Brasília: MS, 2010.

BRASIL. Ministério da Saúde. Envelhecimento e saúde da pessoa idosa. Brasília: MS, 2007.

BRASIL. Ministério da Saúde. Estratégias para o cuidado da pessoa com doença crônica: hipertensão arterial sistêmica. Brasília: MS, 2013.

BRASIL. Ministério da Saúde. Plano de ações estratégicas para o enfrentamento das doenças crônicas não transmissíveis (DCNT) no Brasil: 2011-2022. Brasília: MS, 2011.

BRASIL. Ministério da Saúde. Vigilância de fatores de risco e proteção para doenças crônicas por inquérito telefônico. Brasília: MS, 2010.

BRASIL. Ministério da Saúde. Vigitel Brasil 2011: Vigilância de fatores de risco e proteção para doenças crônicas por inquérito telefônico. Brasília: MS, 2012.

DOURADO, C. S. M. C.; OLIVEIRA, J. S.; LEADEBAL, O. D. C. P.; SILVIA, G. R. F.. Adesão ao tratamento de idosos com hipertensão em uma unidade básica de saúde de João Pessoa. Estado da Paraíba, Maringá, v.33, n.1, p.9-17, 2011.

IBGE. Instituto Brasileiro de Geografia e Estatística. Projeção da População. Rio de Janeiro: IBGE, 2018.

IBGE. Instituto Brasileiro de Geografia e Estatística. Um Panorama da Saúde no Brasil: Acesso e utilização dos serviços, condições de saúde e fatores de risco e proteção à saúde 2008. Rio de Janeiro: IBGE, 2010.

LIBERMAN, A.. Aspectos epidemiológicos e o impacto clinico da hipertensão no indivíduo idoso. Revista Brasileira de Hipertensão, v.14, p.17-20, 2007.

MENDES, G. S.; MORAES, C. F.; GOMES, L.. Prevalência de hipertensão arterial sistêmica em idosos no Brasil entre 2006 e 2010. Revista Brasileira de Medicina de Família e Comunidade, Rio de Janeiro, v.9, n.32, p.273-278, 2014.

SANTOS, J. C.; MOREIRA, T. M. M.. Fatores de risco e complicações em hipertensos/diabéticos de uma regional sanitária do nordeste brasileiro. Revista da Escola Enfermagem da USP, v.46, n.5, p.1125-1132, 2012.

SBC. Sociedade Brasileira de Cardiologia. Diretrizes Brasileiras de Hipertensão. Arquivos Brasileiros de Cardiologia, v.95, p.1-51, 2010.

SOARES, M. M.; SILVA, L. O. L.; DIAS, C. A.; RODRIGUES, S. M.; MACHADO, C. J.. Adesão do idoso ao tratamento da hipertensão arterial sistêmica: revisão integrativa. Cogitare Enfermagem, v.17, n.1, p.144-150, 2012.

SUELY, I. C.; ELIZABETH, B.; MARIA, F. B. N. A. C.; NELIZE, G. R. N.; RUBIA, A. A.. Senescência e senilidade: novo paradigma na Atenção Básica de Saúde. Revista de Escola de Enfermagem da USP, v.45, n.2, p.1763-1768, 2011.

A CBPC - Companhia Brasileira de Produção Científica (CNPJ: 11.221.422/0001-03) detém os direitos materiais desta publicação. Os direitos referem-se à publicação do trabalho em qualquer parte do mundo, incluindo os direitos às renovações, expansões e disseminações da contribuição, bem como outros direitos subsidiários. Todos os trabalhos publicados eletronicamente poderão posteriormente ser publicados em coletâneas impressas sob coordenação da Sustenere Publishing, da Companhia Brasileira de Produção Científica e seus parceiros autorizados. Os (as) autores (as) preservam os direitos autorais, mas não têm permissão para a publicação da contribuição em outro meio, impresso ou digital, em português ou em tradução. 